Article

\title{
Finite-Time Synchronization of Chaotic Complex Networks with Stochastic Disturbance
}

\author{
Liangliang Li and Jigui Jian* \\ College of Science, China Three Gorges University, Yichang, 443002, China; \\ E-Mail: lilianglianglll@126.com \\ * Author to whom correspondence should be addressed; E-Mail: jiguijian@ @ctgu.edu.cn; \\ Tel./Fax: +86-0717-6392370.
}

Academic Editors: Guanrong Chen, C.K. Michael Tse, Mustak E. Yalcin, Hai Yu and Mattia Frasca

Received: 16 October 2014 / Accepted: 22 December 2014 / Published: 30 December 2014

\begin{abstract}
This paper is concerned with the problem of finite-time synchronization in complex networks with stochastic noise perturbations. By using a novel finite-time $\mathscr{L}$-operator differential inequality and other inequality techniques, some novel sufficient conditions are obtained to ensure finite-time stochastic synchronization for the complex networks concerned, where the coupling matrix need not be symmetric. The effects of control parameters on synchronization speed and time are also analyzed, and the synchronization time in this paper is shorter than that in the existing literature. The results here are also applicable to both directed and undirected weighted networks without any information of the coupling matrix. Finally, an example with numerical simulations is given to demonstrate the effectiveness of the proposed method.
\end{abstract}

Keywords: chaotic complex networks; finite-time synchronization; stochastic synchronization; $\mathscr{L}$-operator differential inequality; stochastic disturbance

\section{Introduction}

In recent years, complex networks have been shown to exist in many different areas in the real word [1], such as Internet networks, the Word Wide Web, food chains, relationship networks, and so on. A complex network is composed of a set of interconnected nodes, where the nodes and connections can represent anything. According to different ways of connections and whether there are 
weights or not between nodes, we can get different kinds of complex networks, such as undirected unweighted networks, directed weighted network, etc. However, the network structure facilitates and constrains the network dynamical behaviors. The complex nature of the networks results in a series of important research problems. In particular, one significant and interesting issue is the synchronization problem of dynamics system [2]. Thus, more and more studies have been devoted to discuss synchronization phenomena in small-world and scale-free networks in recent years [3-13]. The synchronization of complex dynamical networks can be divided into the synchronization of complex networks with [4-6,8-12] or without [13] control inputs. Until now, in order to treat the synchronization problem for complex networks, several control schemes, such as state observer-based control [5], impulsive control [6], adaptive control [8,9], pinning control [10,11], sliding mode control and intermittent control [12], were applied. However, many real-world networks, such as biological networks, are time-varying networks, and also, metabolic networks are neither completely regular nor completely random networks. Lü and Chen [3] introduced a time varying model and studied its synchronization problems. In [7], Tan et al. showed that the heat heterogeneity mainly determines the impact of network structure on evolutionary dynamics on complex networks.

Meanwhile, signals transmitted between subsystems of complex networks are unavoidably subjected to stochastic perturbations from the environment [14], which may cause information contained in these signals to be lost. Stochastic systems have received increasing attention and played an important role in many scientific and engineering applications [14,15]. In [16], Karimi considered the $H_{\infty}$ synchronization of master-slave time-delay systems with Markovian jumping parameters by the sliding mode approach. Furthermore, Karimi and Gao et al. [17-19] extended the model to a complex model with uncertain or fuzzy rules. Some $H_{\infty}$ synchronization criteria of complex system are derived for both delay-independent and the delay-dependent exponential stability of the error state. All of the methods mentioned [14,15,17-19] have been used to guarantee the asymptotic, exponentially $H_{\infty}$ stability of the synchronization error dynamics. However, in a practical engineering process, the networks might always be expected to synchronize as quickly as possible [20-25], that is the finite-time synchronization. Finite-time synchronization means the optimality in convergence time. Moreover, the finite-time control techniques have demonstrated better robustness and disturbance rejection properties. In [20,21], the authors investigated the finite-time chaos synchronization. Liu et al. [22] considered the finite-time stabilization for a class of neural networks with discontinuous activations. Sun et al. [23] discussed finite-time stochastic outer synchronization between two complex dynamical networks. In [24], the authors investigated the finite-time stochastic synchronization of complex networks. As far as we know, there are few other researchers that have considered finite-time synchronization in stochastic complex system.

As discussions above, in this paper, we will consider finite-time synchronization in complex networks with stochastic noise perturbations. Some novel sufficient conditions will be obtained for finite-time stochastic synchronization in the complex networks. The results here are different from that in [24], where Yang et al. considered the finite-time stochastic synchronization problem for complex networks by general finite-time stability theory. Here, we will construct a new finite-time $\mathscr{L}$-operator differential inequality and combine it with Lyapunov function and other inequality techniques to investigate finite-time synchronization for the complex networks concerned. It is clear that the controller here can 
shorten the synchronization time, and the synchronization time is shorter than that in [24]. Hence, the results here improve existing ones in the estimates of convergence time. Finally, a numerical simulation shows the effectiveness of the synchronization control scheme.

This paper is organized as follows. In Section 2, some preliminaries are described. The main results are given in Section 3. In Section 4, one numerical example is provided to illustrate the effectiveness of the proposed results. Concluding remarks are stated in Section 5.

\section{Preliminaries}

Throughout this paper, let $R_{+}=[0,+\infty) . R^{n}$ is the $n$-dimensional Euclidean space. $R^{n \times m}$ denotes the set of $n \times m$ real matrix. $\operatorname{Tr}(X)$ denotes its trace when $X$ is square. $\mathbb{E}(x)$ denotes the expectation of stochastic variable $x$. The norm $\|\cdot\|$ of a vector $x$ is defined as $\|x\|=\left(x^{T} x\right)^{\frac{1}{2}}$. $I_{n}$ denotes the $n$-dimensional identity matrix. $\otimes$ stands for the notation of Kronecker product. For a general stochastic system:

$$
d x(t)=g(t, x(t)) d t+\sigma(t, x(t)) d \omega(t)
$$

where $\omega(t)$ is an $n$-dimensional Brownian motion defined on a complete probability space $(\Omega, \mathscr{F}, P)$, we have $\mathbb{E} \omega(t)=0$. An operator $\mathscr{L}$ is defined by:

$$
\mathscr{L} V(t, x)=V_{t}(t, x)+V_{x}(t, x) g(t, x)+\frac{1}{2} \operatorname{Tr}\left[\sigma(t, x(t))^{T} V_{x x}(t, x) \sigma(t, x(t))\right],
$$

where $V_{t}(t, x)=\frac{\partial V(t, x)}{\partial t}, V_{x}(t, x)=\left(\frac{\partial V(t, x)}{\partial x_{1}}, \frac{\partial V(t, x)}{\partial x_{2}}, \cdots, \frac{\partial V(t, x)}{\partial x_{n}}\right), V_{x x}(t, x)=\left(\frac{\partial^{2} V(t, x)}{\partial x_{i} \partial x_{j}}\right)_{n \times n}$.

Now, we consider complex networks consisting of $N$ identical nodes with linearly diffusive couplings, in which each node is an $n$-dimensional dynamical system described by the following equation:

$$
d x_{i}(t)=\left[f\left(x_{i}(t)\right)+c_{0} \sum_{j=1}^{N} g_{i j} \Gamma x_{j}(t)+u_{i}(t)\right] d t+\sigma_{i}(x(t)) d \omega_{i}(t), \quad i=1,2, \cdots, N,
$$

where $x_{i}(t)=\left(x_{i 1}(t), x_{i 2}(t), \cdots, x_{i n}(t)\right)^{T} \in R^{n}$ is the state vector of the $i$-th node, $f: R^{n} \rightarrow R^{n}$ is a smooth nonlinear vector field and $c_{0}$ is the coupling strength. $\Gamma \in R^{n \times n}$ is the inner coupling matrix. $G=\left(g_{i j}\right)_{N \times N}$ is a coupling matrix, where $g_{i j}$ is defined as follows: if there is a coupling link from node $i$ to node $j(i \neq j)$, then $g_{i j}>0$; otherwise, $g_{i j}=0$. In addition, assume that $G$ are diffusive matrices satisfying:

$$
g_{i i}=-\sum_{j=1, j \neq i}^{N} g_{i j}, \quad i=1,2, \cdots, N
$$

$\sigma_{i}(x(t)): R^{n} \rightarrow R^{n \times n}$ is called the noisy intensity matrix. $\omega_{i}(t)=\left(\omega_{i 1}(t), \omega_{i 2}(t), \cdots, \omega_{i n}(t)\right)^{T}$ is a vector Wiener process. $u_{i}(t) \in R^{n}$ is a feedback controller. The initial state vector value is $x(0)=\left(x_{1}^{T}(0), x_{2}^{T}(0) \cdots, x_{N}^{T}(0)\right)^{T}$.

Assumption 1. There exists a nonnegative constant $\omega$, such that $f$ satisfies the following inequality:

$$
(x-y)^{T}[f(x)-f(y)] \leq \omega(x-y)^{T}(x-y)
$$

for any $x, y \in R^{n}$. 
Remark 1. In fact, it is easy to verify that the class of systems in the form of Assumption 1 includes almost all of the well-known chaotic systems, such as the Lorenz system, Chen system and Chua's circuit. Generally speaking, any functions satisfying the Lipschitz condition [23-25] can guarantee Assumption 1.

Assume that $s(t) \in R^{n}$ is a solution of an isolated node and satisfies:

$$
d s(t)=f(s(t)) d t
$$

$s(t)$ can be an equilibrium point, a periodic orbit, an aperiodic orbit or even a chaotic orbit in the phase space.

The diffusive couplings mean that coupled networks (2) are decoupled when the systems are synchronized. Therefore, the coupling terms satisfy $\sum_{j=1}^{N} g_{i j} \Gamma s(t)=0$. We denote:

$$
\begin{gathered}
\sigma_{i}(x(t))=\sigma_{i}\left(x_{i}(t), x_{2}(t), \cdots, x_{N}(t)\right), \quad i=1,2, \cdots, N \\
\sigma_{i}(s(t))=\sigma_{i}(s(t), s(t), \cdots, s(t)), \quad i=1,2, \cdots, N .
\end{gathered}
$$

Additionally, $\sigma_{i}(s(t), s(t), \cdots, s(t))=0_{n \times n}, 0_{n \times n}$ denotes the zero matrix of $n \times n$-dimension. Define the error vectors as:

$$
e_{i}(t)=x_{i}(t)-s(t), \quad i=1,2, \cdots, N .
$$

From Equations (2), (3), (5) and (6), we can obtain the following error systems:

$$
d e_{i}(t)=\left[f\left(x_{i}(t)\right)-f(s(t))+c_{0} \sum_{j=1}^{N} g_{i j} \Gamma e_{j}(t)+u_{i}(t)\right] d t+\tilde{\sigma}_{i}(e(t)) d \omega_{i}(t),
$$

where $\tilde{\sigma}_{i}(e(t))=\sigma_{i}(x(t))-\sigma_{i}(s(t))$. In order to achieve finite-time synchronization, the controllers are designed as follows:

$$
u_{i}(t)=-\eta e_{i}(t)-\xi \operatorname{sign}\left(e_{i}(t)\right)\left|e_{i}(t)\right|^{\zeta},
$$

where $\eta>0, \xi>0$ are constants, which can be adjusted, and the real number $\zeta$ satisfies $0<\zeta<1 . \quad\left|e_{i}(t)\right|^{\zeta}=\left(\left|e_{i 1}(t)\right|^{\zeta},\left|e_{i 2}(t)\right|^{\zeta}, \cdots,\left|e_{i n}(t)\right|^{\zeta}\right)^{T}, \operatorname{sign}\left(e_{i}(t)\right)=$ $\operatorname{diag}\left(\operatorname{sign}\left(e_{i 1}(t)\right), \operatorname{sign}\left(e_{i 2}(t)\right), \cdots, \operatorname{sign}\left(e_{i n}(t)\right)\right)$.

For the noise intensity function, we also need the following assumption:

Assumption 2. The noise intensity function $\tilde{\sigma}_{i}(e(t))$ satisfies the Lipschitz condition, and there exist positive constants $r_{i j}$, such that:

$$
\operatorname{Tr}\left(\left(\tilde{\sigma}_{i}(e(t))\right)^{T} \tilde{\sigma}_{i}(e(t))\right) \leq \sum_{j=1}^{N} r_{i j}\left\|x_{j}(t)-s(t)\right\|^{2},
$$

where $i=1,2, \cdots, N, \tilde{\sigma}_{i}(e(t))=\sigma_{i}(x(t))-\sigma_{i}(s(t))$.

Definition 1. Complex networks (2) are said to be stochastically synchronized in finite time, if for a suitable designed feedback controllers (8), there exists a constant $T_{s}>0$, such that:

$$
\lim _{t \rightarrow T_{s}} \mathbb{E}\left\|x_{i}(t)-s(t)\right\|=0
$$

and $\left\|x_{i}(t)-s(t)\right\| \equiv 0$ for $t>T_{s}, i=1,2, \cdots, N$, where $T_{s}$ depends on the initial state vector value. 
Lemma 1. (Jensen inequality.) If $a_{1}, a_{2}, \cdots, a_{n}$ are positive numbers and $0<r<p$, then:

$$
\left(\sum_{i=1}^{n} a_{i}^{p}\right)^{1 / p} \leq\left(\sum_{i=1}^{n} a_{i}^{r}\right)^{1 / r} .
$$

Lemma 2. [26] Assume that $\alpha(\cdot): R \rightarrow R$ and $\beta(\cdot): R^{n} \rightarrow R$ are two smooth functions, and $x(t)$ is the solution of network (1), then the following equation holds.

$$
\mathscr{L}[\alpha(\beta(x))]=\frac{d \alpha}{d \beta} \mathscr{L}[\beta(x)]+\frac{1}{2} \frac{d^{2} \alpha}{d \beta^{2}} \operatorname{Tr}\left[\left(\frac{\partial \beta}{\partial x} g\right)^{T}\left(\frac{\partial \beta}{\partial x} g\right)\right] .
$$

Lemma 3. Assume that System (1) has a unique global solution. If there exist a positive definite, twice continuous differential and radially unbounded Lyapunov function $V(x(t)): R^{n} \rightarrow R_{+}$and real numbers $k_{1}>0, k_{2}>0$ and $0<\eta<1$, such that:

$$
\mathscr{L} V(x(t)) \leq-k_{1} V^{\eta}(x(t))-k_{2} V(x(t))
$$

$\forall x(t) \in U \backslash\{0\}$, then the origin of System (1) is globally stochastically finite-time stable, and the settling time function $T_{s}$ satisfies $\mathbb{E}\left[T_{s}\right] \leq \frac{\ln \left(1+\frac{k_{2}}{k_{1}} V^{1-\eta}(x(0))\right)}{k_{2}(1-\eta)}$.

Proof. Since $\mathscr{L} V(x(t)) \leq-k_{1} V^{\eta}(x(t))-k_{2} V(x(t)) \leq 0$, we can easily conclude that the origin is globally stable in probability. Next, we will prove that the origin is globally stable in finite time.

We define a function:

$$
\alpha(V(x(t)))=\int_{0}^{V(x(t))} \frac{1}{k_{1} v^{\eta}+k_{2} v} d v .
$$

Since $x(0) \neq 0$, there must exist $k \in N=:\{1,2, \cdots\}$, such that $\frac{1}{k}<|x(0)|<k$.

Define an increasing stop time sequence as follows:

$$
\tau_{k}=\inf \left\{t \geq 0:\|x(t, 0)\| \notin\left(\frac{1}{k}, k\right)\right\} .
$$

When $t \leq \tau_{k}$, based on Lemma 2, we have:

$$
\begin{aligned}
\mathscr{L}[\alpha(V(x(t)))]= & \frac{\mathscr{L}[V(x(t))]}{k_{1} V^{\eta}(x(t))+k_{2} V(x(t))} \\
& -\frac{1}{2} \frac{\left(k_{1} \eta V^{\eta-1}(x(t))+k_{2}\right)}{\left(k_{1} V^{\eta}(x(t))+k_{2} V(x(t))\right)^{2}} \operatorname{Tr}\left[\left(\frac{\partial V}{\partial x} g\right)^{T}\left(\frac{\partial V}{\partial x} g\right)\right] .
\end{aligned}
$$

From Condition (11), it follows that:

$$
\mathscr{L}[\alpha(V(x(t)))] \leq-1
$$

Combining with the theory of stochastic differential equations [26], then:

$$
\left.E[\alpha(V(x(t)))]\right|_{0} ^{k \wedge \tau_{k}}=E \int_{0}^{k \wedge \tau_{k}} \mathscr{L}[\alpha(V(x(s)))] d s,
$$

where $k \wedge \tau_{k}$ denotes the minimum of $k$ and $\tau_{k}$.

Therefore,

$$
\mathbb{E}\left[\alpha\left(V\left(x\left(k \wedge \tau_{k}\right)\right)\right)\right]-\mathbb{E}[\alpha(V(x(0)))] \leq-\mathbb{E}\left[k \wedge \tau_{k}\right]
$$


Considering $\mathbb{E}\left[\alpha\left(V\left(x\left(k \wedge \tau_{k}\right)\right)\right)\right] \geq 0$, so we get:

$$
\mathbb{E}\left[k \wedge \tau_{k}\right] \leq \mathbb{E}[\alpha(V(x(0)))]=\alpha(V(x(0))) .
$$

Let $k \rightarrow+\infty$; then, we have $k \wedge \tau_{k} \rightarrow T_{s}$ a.s. By the well-known Fatou Lemma, we have:

$$
\begin{aligned}
\mathbb{E}\left(T_{s}\right) & \leq \alpha(V(x(0)))=\int_{0}^{V(x(0))} \frac{1}{k_{1} v^{\eta}+k_{2} v} d v \\
& \leq \frac{\ln \left(1+\frac{k_{2}}{k_{1}} V^{1-\eta}(x(0))\right)}{k_{2}(1-\eta)}<+\infty
\end{aligned}
$$

which implies $T_{s}<+\infty$ a.s. This completes the proof.

Remark 2. In [26], Chen and Jiao considered the finite-time stability theorem of stochastic nonlinear system. They offered $\mathscr{L} V(x(t)) \leq-k_{1} V^{\eta}(x(t))$ as a finite-time $\mathscr{L}$-operator differential inequality, and the finite time is estimated by $\mathbb{E}\left(T_{0}\right) \leq \frac{V^{1-\eta}(x(0))}{k_{1}(1-\eta)}<+\infty$. Here, we construct a new finite-time $\mathscr{L}$-operator and re-estimate the convergence time by $\mathbb{E}\left(T_{s}\right) \leq \frac{\ln \left(1+\frac{k_{2}}{k_{1}} V^{1-\eta}(x(0))\right)}{k_{2}(1-\eta)}$. Obviously, $\frac{\ln \left(1+\frac{k_{2}}{k_{1}} V^{1-\eta}(x(0))\right)}{k_{2}(1-\eta)} \leq \frac{V^{1-\eta}(x(0))}{k_{1}(1-\eta)}$. Therefore, the results here improve existing results [26] in estimates of convergence time.

\section{Main Results}

In the following, we will give the finite-time synchronization criteria of stochastic complex networks (2).

Theorem 1. Let Assumptions 1 and 2 hold. If there exists a positive constant $\rho$ satisfying:

$$
(\omega-\eta) I_{N n}+\frac{c_{0}}{2}\left(G+G^{T}\right) \otimes \Gamma+\frac{1}{2} R_{N} \otimes I_{N} \leq-\rho I_{N n},
$$

then control dynamical networks (2) with the set of controllers (8) is finite-time stochastic synchronized. Moreover, complex networks (2) are synchronized in a finite time $T_{s}$ :

$$
\mathbb{E}\left(T_{s}\right) \leq \frac{\ln \left(1+\frac{\rho}{2^{\frac{1+\zeta}{2}} \xi} V^{\frac{1-\zeta}{2}}(e(0))\right)}{\rho\left(\frac{1-\zeta}{2}\right)}
$$

where $V(e(0))=\frac{1}{2} \sum_{i=1}^{N} e_{i}^{T}(0) e_{i}(0), \sum_{i=1}^{N} r_{i j}=r_{j}$ and $R_{N}=\operatorname{diag}\left(r_{1}, r_{2}, \cdots, r_{N}\right) . I_{N n}$ is $a(N \times n) \times$ $(N \times n)$-dimensional identity matrix.

Proof. Construct the following Lyapunov function:

$$
V(t)=V(x(t))=\frac{1}{2} \sum_{i=1}^{N} e_{i}^{T}(t) e_{i}(t) .
$$


Note $e(t)=\left(e_{1}^{T}(t), e_{2}^{T}(t), \cdots, e_{N}^{T}(t)\right)^{T}$; by the well-known $\mathscr{L}$-operator given by the Itô formula [15,23]. Computing the time derivative of $V(t)$ along the trajectories of (7) and combining with Assumption 1, we can obtain:

$$
\begin{aligned}
\mathscr{L} V(t)= & \sum_{i=1}^{N} e_{i}^{T}(t) \dot{e}_{i}(t)+\frac{1}{2} \sum_{i=1}^{N} \operatorname{Tr}\left(\left(\tilde{\sigma}_{i}(e(t))\right)^{T} \tilde{\sigma}_{i}(e(t))\right) \\
\leq & \sum_{i=1}^{N} e_{i}^{T}(t)\left[f\left(x_{i}(t)\right)-f(s(t))+c_{0} \sum_{j=1}^{N} g_{i j} \Gamma e_{j}(t)+u_{i}(t)\right]+\frac{1}{2} \sum_{i=1}^{N} \sum_{j=1}^{N} r_{i j}\left\|e_{j}(t)\right\|^{2} \\
\leq & \omega \sum_{i=1}^{N} e_{i}^{T}(t) e_{i}(t)+c_{0} \sum_{i=1}^{N} \sum_{j=1}^{N} g_{i j} e_{i}^{T}(t) \Gamma e_{j}(t)-\sum_{i=1}^{N} \eta e_{i}^{T}(t) e_{i}(t) \\
& -\xi \sum_{i=1}^{N} e_{i}^{T}(t) \operatorname{sign}\left(e_{i}(t)\right)\left|e_{i}(t)\right|^{\zeta}+\frac{1}{2} \sum_{j=1}^{N} \sum_{i=1}^{N} r_{i j}\left\|e_{j}(t)\right\|^{2} \\
\leq & e^{T}(t)\left[(\omega-\eta) I_{N n}+\frac{c_{0}}{2}\left(G+G^{T}\right) \otimes \Gamma+\frac{1}{2} R_{N} \otimes I_{N}\right] e(t) \\
& -\xi \sum_{i=1}^{N} e_{i}^{T}(t) \operatorname{sign}\left(e_{i}(t)\right)\left|e_{i}(t)\right|^{\zeta} .
\end{aligned}
$$

Owing to:

$$
\sum_{i=1}^{N} e_{i}^{T}(t) \operatorname{sign}\left(e_{i}(t)\right)\left|e_{i}(t)\right|^{\zeta}=\sum_{i=1}^{N} \sum_{j=1}^{n}\left|e_{i j}(t)\right|^{1+\zeta}
$$

according to Lemma 1, we get:

$$
\left(\sum_{i=1}^{N} \sum_{j=1}^{n}\left|e_{i j}(t)\right|^{1+\zeta}\right)^{\frac{1}{1+\zeta}} \geq\left(\sum_{i=1}^{N} \sum_{j=1}^{n}\left|e_{i j}(t)\right|^{2}\right)^{\frac{1}{2}} .
$$

Hence, we have:

$$
\sum_{i=1}^{N} \sum_{j=1}^{n}\left|e_{i j}(t)\right|^{1+\zeta} \geq\left(\sum_{i=1}^{N} \sum_{j=1}^{n}\left|e_{i j}(t)\right|^{2}\right)^{\frac{1+\zeta}{2}}=\left(\sum_{i=1}^{N} e_{i}^{T}(t) e_{i}(t)\right)^{\frac{1+\zeta}{2}}
$$

Therefore,

$$
\mathscr{L} V(t) \leq-2^{\frac{1+\zeta}{2}} \xi V^{\frac{1+\zeta}{2}}(t)-\rho V(t)
$$

Combining with Lemma 3, $V(t)$ converges to zero in a finite time, and the finite time can be estimated by:

$$
\mathbb{E}\left(T_{s}\right) \leq \frac{\ln \left(1+\frac{\rho}{2^{\frac{1+\zeta}{2}} \xi} V^{\frac{1-\zeta}{2}}(e(0))\right)}{\rho\left(\frac{1-\zeta}{2}\right)} .
$$

Hence, the error vectors $e_{i}(t)$ for $i=1,2, \cdots, N$ converge to zero within $T_{s}$. Consequently, the coupled complex networks (2) with the controllers (8) are finite-time stochastic synchronized in the finite time $T_{s}$.

This completes the proof.

Remark 3. To the best of our knowledge, there are some results concerning finite-time synchronization [23-25]. Compared with the existing results, there are two obvious advantages: 
(1) The coupling matrix $G$ here need not be symmetric [25]. (2) Our results can greatly shorten the synchronization time than that obtained by the method in [24]. In most cases, to achieve faster synchronization in control systems, many authors generally make use of the methods in [24], where the finite time is estimated by $\tilde{T}_{s} \leq \frac{\left.V^{1-\eta}\left(x_{0}\right)\right)}{l(1-\eta)}$. In this paper, combining with Lemma 3, the finite time is estimated by $\mathbb{E}\left(T_{s}\right) \leq \frac{\ln \left(1+\frac{h}{l} V^{1-\eta}\left(x_{0}\right)\right)}{h(1-\eta)}$. It is easy to obtain that $\frac{\left.V^{1-\eta}\left(x_{0}\right)\right)}{l(1-\eta)}>\frac{\ln \left(1+\frac{h}{l} V^{1-\eta}\left(x_{0}\right)\right)}{h(1-\eta)}$. It is clear that the shorter time can be obtained when the term $h V(x)$ is added. However, owing to increasing the term $h V(x)$, the condition (12) guaranteeing the finite time synchronization here is stronger. Next, through the frequently-used methods as in [24], we will make a clear comparison in the following Corollary 1.

If using the method in [24], we can obtain:

Corollary 1. Let Assumptions 1 and 2 hold. If the following inequality holds,

$$
(\omega-\eta) I_{N n}+\frac{c_{0}}{2}\left(G+G^{T}\right) \otimes \Gamma+\frac{1}{2} R_{N} \otimes I_{N} \leq 0,
$$

then control dynamical networks (2) with the set of controllers (8) are finite-time stochastic synchronized. Moreover, complex networks (2) are synchronized in a finite time $\tilde{T}_{s}$ :

$$
\tilde{T}_{s} \leq \frac{V^{\frac{1-\zeta}{2}}(e(0))}{\xi\left(\frac{1-\zeta}{2}\right)}
$$

where $V(e(0))=\frac{1}{2} \sum_{i=1}^{N} e_{i}^{T}(0) e_{i}(0), \sum_{i=1}^{N} r_{i j}=r_{j}$ and $R_{N}=\operatorname{diag}\left(r_{1}, r_{2}, \cdots, r_{N}\right)$.

Remark 4. Obviously, from Theorem 1 and Corollary 1, it is easy to calculate that $\frac{V^{\frac{1-\zeta}{2}}(e(0))}{\xi\left(\frac{1-\zeta}{2}\right)}>$ $\frac{\ln \left(1+\frac{\rho}{2^{\frac{1+\zeta}{2}} \xi} V^{\frac{1-\zeta}{2}}(e(0))\right)}{\rho\left(\frac{1-\zeta}{2}\right)}$. Therefore, a shorter time can be estimated by employing the method in this paper rather than the frequently-used methods in [24].

Remark 5. When $\sigma_{i}(x(t))$ is an $n \times n$-dimensional zero matrix, then complex networks (2) turn into the following complex networks without stochastic perturbation:

$$
\dot{x}_{i}(t)=f\left(x_{i}(t)\right)+c_{0} \sum_{j=1}^{N} g_{i j} \Gamma x_{j}(t)+u_{i}(t), \quad i=1, \cdots, N
$$

By means of Theorem 1, the following results are obvious; hence, we omit the proof here.

Corollary 2. Let Assumption 1 holds. Suppose that there exists a positive constant $\rho$ satisfying:

$$
(\omega-\eta) I_{N n}+\frac{c_{0}}{2}\left(G+G^{T}\right) \otimes \Gamma \leq-\rho I_{N n},
$$

then control dynamical networks (19) with the set of controllers (8) are finite-time synchronized. Moreover, complex networks (19) are synchronized in a finite time $T_{s}$ :

$$
T_{s} \leq \frac{\ln \left(1+\frac{\rho}{2^{\frac{1+\zeta}{2}} \xi} V^{\frac{1-\zeta}{2}}(e(0))\right)}{\rho\left(\frac{1-\zeta}{2}\right)}
$$

where $V(e(0))=\frac{1}{2} \sum_{i=1}^{N}\left\|e_{i}(0)\right\|^{2}$. 


\section{Example}

In order to show the effectiveness of the proposed method, we present one numerical example, which is finite-time synchronized for complex networks with $N$ identical nodes, where each node is Chua's chaotic circuit, and it has a chaotic attractor displayed in Figure 1.

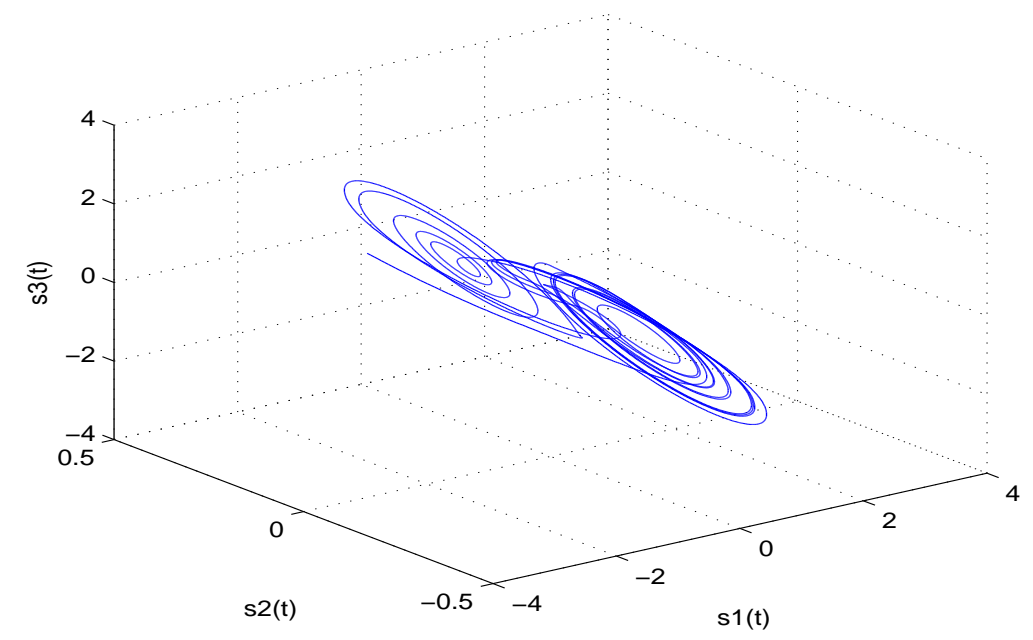

Figure 1. The chaotic behavior of Chua's circuit with initial condition $s(0)=(0.1,0.5,-0.7)^{T}$.

The complex networks consisting of four nodes is described by:

$$
d x_{i}(t)=\left[f\left(x_{i}(t)\right)+c_{0} \sum_{j=1}^{4} g_{i j} \Gamma x_{j}(t)+u_{i}(t)\right] d t+\sigma_{i}(x(t)) d \omega_{i}(t), \quad i=1,2,3,4,
$$

where:

$$
\begin{gathered}
f\left(x_{i}(t)\right)=\left(\begin{array}{c}
9\left(x_{i 2}(t)-h\left(x_{i 1}(t)\right)\right) \\
x_{i 1}(t)-x_{i 2}(t)+x_{i 3}(t) \\
14.28 x_{i 2}(t)
\end{array}\right), \\
h\left(x_{i 1}(t)\right)=-\frac{1}{7} x_{i 1}(t)-\frac{3}{14}\left(\left|x_{i 1}(t)+1\right|-\left|x_{i 1}(t)-1\right|\right) .
\end{gathered}
$$

It is noted that the Lipschitz constant [2] of Chua's circuit is $\omega=5$, where $c_{0}=2$. Here, the initial conditions of each nodes are chosen:

$$
\begin{gathered}
x_{1}(0)=(-0.4,0.4,-0.3)^{T}, x_{2}(0)=(-0.6,0.07,-0.4)^{T}, \\
x_{3}(0)=(0.2,0.6,-0.7)^{T}, x_{4}(0)=(0.8,0,-0.1)^{T}, \\
s(0)=(0.1,0.5,-0.7)^{T} .
\end{gathered}
$$

Coupling matrix $G$ and $\Gamma$ are given by: 


$$
G=\left(\begin{array}{cccc}
-3 & 1 & 1 & 1 \\
1 & -3 & 1 & 1 \\
0 & 1 & -2 & 1 \\
0 & 1 & 1 & -2
\end{array}\right), \Gamma=\left(\begin{array}{lll}
1 & 0 & 0 \\
0 & 1 & 0 \\
0 & 0 & 1
\end{array}\right)
$$

Take $\sigma_{i}(x(t))=\operatorname{diag}\left(x_{i 1}-x_{i+1,1}, x_{i 2}-x_{i+1,2}, x_{i 3}-x_{i+1,3}\right), x_{5}(t)=x_{1}(t), i=1,2,3,4$. We can verify that Assumption 2 is satisfied. Since:

$$
\begin{aligned}
\left\|\sigma_{i}(x(t))-\sigma_{i}(s(t))\right\|^{2} & =\left(x_{i 1}-x_{i+1,1}\right)^{2}+\left(x_{i 2}-x_{i+1,2}\right)^{2}+\left(x_{i 3}-x_{i+1,3}\right)^{2} \\
& =\left(e_{i 1}-e_{i+1,1}\right)^{2}+\left(e_{i 2}-e_{i+1,2}\right)^{2}+\left(e_{i 3}-e_{i+1,3}\right)^{2} \\
& \leq 2\left(\left\|e_{i}\right\|^{2}+\left\|e_{i+1}\right\|^{2}\right) \\
& \leq 2\left(\left\|e_{i}\right\|+\left\|e_{i+1}\right\|\right)^{2} .
\end{aligned}
$$

We can obtain:

$$
\operatorname{Tr}\left(\left(\tilde{\sigma}_{i}(e(t))\right)^{T} \tilde{\sigma}_{i}(e(t))\right) \leq 2\left(\left\|e_{i}\right\|^{2}+\left\|e_{i+1}\right\|^{2}\right), \quad i=1,2,3,4, e_{5}(t)=e_{1}(t)
$$

Therefore, Assumption 2 is satisfied.

The controllers are chosen as:

$$
u_{i}(t)=-9 e_{i}(t)-5 \operatorname{sign}\left(e_{i}(t)\right)\left|e_{i}(t)\right|^{0.8}, \quad i=1,2,3,4 .
$$

In order to show the original behavior of complex networks (22), the trajectories of the error signals without the controller are depicted in Figure 2.

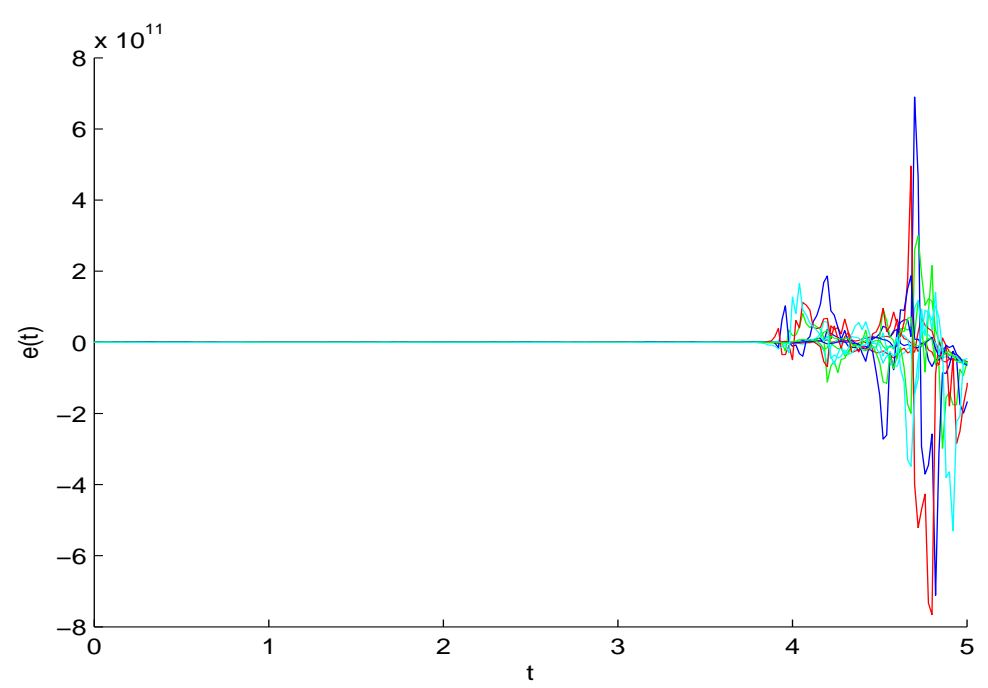

Figure 2. Error signals without controllers.

There exists a positive constant $\rho=1.5$, such that:

$$
(\omega-\eta) I_{N n}+\frac{c_{0}}{2}\left(G+G^{T}\right) \otimes \Gamma+\frac{1}{2} R \otimes I_{N} \leq-\rho I_{N n} .
$$


Therefore, it follows from Theorem 1 that complex networks (22) are finite-time synchronized with the controllers (24). Figure 3 shows the time responses of the error variables $e_{i 1}(t), e_{i 2}(t), e_{i 3}(t)$ for $i=1,2,3,4$. It can be seen that the controllers (24) can synchronize complex networks (22) at about $t=0.7 \mathrm{~s}$, which is smaller than $\mathbb{E}\left(T_{s}\right) \leq \frac{\ln \left(1+\frac{\rho}{2^{\frac{1+\zeta}{2}} \xi} V^{\frac{1-\zeta}{2}}(e(0))\right)}{\rho\left(\frac{1-\zeta}{2}\right)}=1.0071 \leq \frac{V^{\frac{1-\zeta}{2}}(e(0))}{\xi\left(\frac{1-\zeta}{2}\right)}=2.0286$. It is obvious that the synchronization time obtained using Theorem 1 is shorter than that obtained by Corollary 1. It is worth pointing out that we were not here concerned with the computational complexity, and the computational burden of our approach is similar to that of the method in [24].
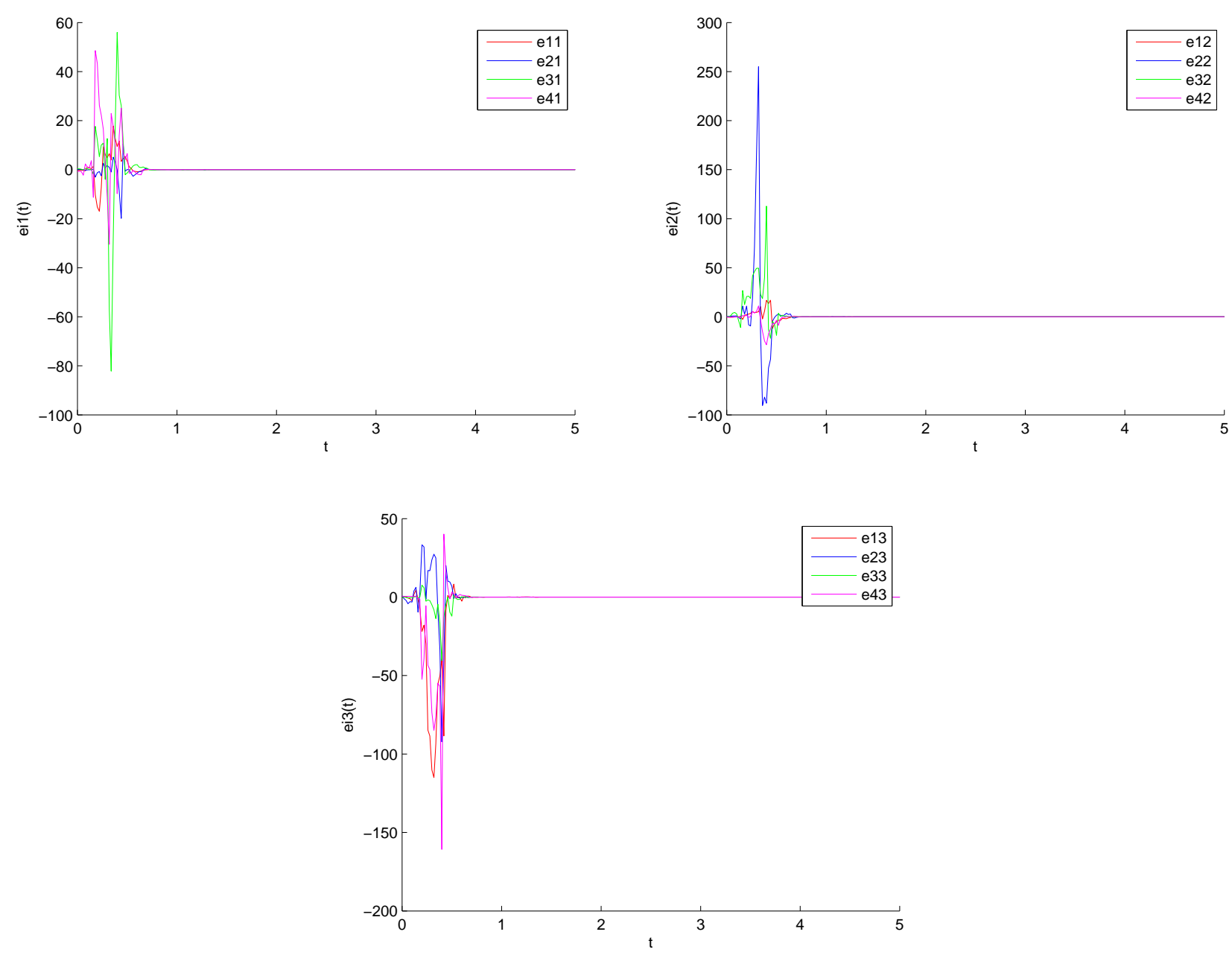

Figure 3. Error signals with controllers (24).

\section{Conclusions}

In this paper, the finite-time synchronization of stochastic complex networks has been studied by finite-time stability theory and inequality techniques. Some sufficient conditions are obtained to ensure finite-time synchronization for the complex networks. The results of this paper are applicable to both directed and undirected weighted networks. In addition, the method in this paper can also be extended to investigate the finite-time synchronization of time-varying networks, such as in [3]. Additionally, under 
appropriate conditions, a further issue is worth addressing the finite-time consensus of the discrete-time multiagent systems, such as $[27,28]$. Finally, one example is given to demonstrate the effectiveness of the proposed method.

\section{Acknowledgments}

The authors are grateful for the support of the National Natural Science Foundation of China (61174216, 61304162, 61273183, 61374028), the Scientific Innovation Team Project of Hubei Provincial Department of Education (T200809) and the Graduate Scientific Research Foundation of China Three Gorges University (2014PY063).

\section{Author Contributions}

Both authors jointly worked on deriving the results and wrote the paper. Both authors have read and approved the final manuscript.

\section{Conflicts of Interest}

The authors declare no conflict of interest.

\section{References}

1. Newman, M.E.J. The structure and function of complex networks. SIAM Rev. 2003, 45, 167-256.

2. Lee, T.H.; Park, J.H.; Ji, D.H.; Lee, S.M. Guaranteed cost synchronization of a complex dynamical network via dynamic feedback control. App. Math. Comput. 2012, 218, 6469-6481.

3. Lü, J.; Chen, G. A time-varying complex dynamical network model and its controlled synchronization criteria. IEEE Trans. Autom. Control 2005, 50, 841-846.

4. Chen, L.; Qu, J.; Chai, Y.; Wu, R.; Qi, G. Synchronization of a class of fractional-order chaotic neural networks. Entropy 2013, 15, 3265-3276.

5. Zhao, M.; Zhang, H.; Wang, Z.; Liang, H. Observer-based lag synchronization between two different complex networks. Commun. Nonlinear Sci. Numer. Simul. 2014, 19, 2048-2059.

6. Zheng, S.; Dong, G.; Bi, Q. Impulsive synchronization of complex networks with non-delayed and delayed coupling. Phys. Lett. A 2009, 373, 4255-4259.

7. Tan, S.; Lü, J. Characterizing the effect of population heterogeneity on evolutionary dynamics on complex networks. Sci. Rep. 2014, 4, doi:10.1038/srep05034.

8. Cao, J.; Lu, J. Adaptive synchronization of neural networks with or without time-varying delay. Chaos 2006, 16, doi:10.1063/1.2178448.

9. Zhang, Q.; Lu, J.; Lü, J.; Tse, C.K. Adaptive feedback synchronization of a general complex dynamical network with delayed nodes. IEEE Trans. Circuits Syst. II 2008, 55, 183-187.

10. Liang, Y.; Wang, X.; Eustace, J. Adaptive synchronization in complex networks with non-delay and variable delay couplings via pinning control. Neurocomputing 2014, 123, 292-298.

11. Yu, W.; Chen, G.; Lu, J.; Kurths, J. Synchronization via pinning control on general complex networks. SIAM J. Control Optim. 2013, 51, 1395-1416. 
12. Sun, H.; Li, N.; Zhao, D.; Zhang, Q. Synchronization of complex networks with coupling delays via adaptive pinning intermittent control. Int. J. Autom. Comput. 2013, 10, 312-318.

13. Li, C.; Chen, G. Synchronization in general complex dynamical networks with coupling delays. Physica A 2004, 343, 263-278.

14. Guo, X.; Li, J. Stochastic synchronization for time-varying complex dynamical networks. Chin. Phys. B 2012, 21, doi:10.1088/1674-1056/21/2/020501.

15. Yang, X.; Cao, J.; Lu, J. Stochastic synchronization of complex networks with nonidentical nodes via hybrid adaptive and impulsive control. IEEE Trans. Circuits. Syst. I 2012, 59, 371-384.

16. Karimi, H.R. A sliding mode approach to $H_{\infty}$ synchronization of master-slave time-delay systems with Markovian jumping parameters and nonlinear uncertainties. J. Frankl. Inst. 2012, 349, 1480-1496.

17. Wang, B.; Shi, P.; Karimi, H.R.; Wang, J. $H_{\infty}$ robust controller design for the synchronization of master-slave chaotic systems with disturbance input. Model. Identif. Control 2012, 33, 27-34.

18. Karimi, H.R.; Gao, H. New delay-dependent exponential $H_{\infty}$ synchronization for uncertain neural networks with mixed time delays. IEEE Trans. Syst. Man Cybern. B 2010, 40, 173-185.

19. Zhao, Y.; Li, B.; Qin, J.; Gao, H.; Karimi, H.R. Consensus and synchronization of nonlinear systems based on a novel fuzzy model. IEEE Trans. Cybern. 2013, 43, 2157-2169.

20. Luo, R.; Wang, Y. Finite-time stochastic combination synchronization of three different chaotic systems and its application in secure communication. Chaos 2012, 22, doi:10.1063/1.3702864.

21. Wang, H.; Han, Z.; Xie, Q.; Zhang, W. Finite-time chaos synchronization of unified chaotic system with uncertain parameters. Commun. Nonlinear Sci. Numer. Simul. 2009, 14, 2239-2247.

22. Liu, X.; Park, J.H.; Jiang, N.; Cao, J. Nonsmooth finite-time stabilization of neural networks with discontinuous activations. Neural Netw. 2014, 52, 25-32.

23. Sun, Y.; Li, W.; Zhao, D. Finite-time stochastic outer synchronization between two complex dynamical networks with different topologies. Chaos 2012, 22, doi:10.1063/1.4731265.

24. Yang, X.; Cao, J. Finite-time stochastic synchronization of complex networks. Appl. Math. Model. 2010, 34, 3631-3641.

25. Li, B. Finite-time synchronization for complex dynamical networks with hybrid coupling and time-varying delay. Nonlinear Dyn. 2014, 76, 1-8.

26. Chen, W.; Jiao, L. Authors' reply to "Comments on 'Finite-time stability theorem of stochastic nonlinear systems"'. Automatica 2011, 47, 1544-1545.

27. Chen, Y.; Lü, J.; Yu, X.; Lin, Z. Consensus of discrete-time second-order multiagent systems based on infinite products of general stochastic matrices. SIAM J. Control Optim. 2013, 51, 3274-3301.

28. Chen, Y.; Lü, J.; Lin, Z. Consensus of discrete-time multi-agent systems with transmission nonlinearity. Automatica 2013, 49, 1768-1775.

(C) 2014 by the authors; licensee MDPI, Basel, Switzerland. This article is an open access article distributed under the terms and conditions of the Creative Commons Attribution license (http://creativecommons.org/licenses/by/4.0/). 